\title{
A Blotto game with Incomplete Information ${ }^{2}$
}

\author{
Tim Adamo, Alexander Matros* \\ University of Pittsburgh, USA
}

\section{A R T I C L E I N F O}

\section{Article history:}

Received 31 July 2008

Received in revised form 13 June 2009

Accepted 22 June 2009

Available online 17 July 2009

\section{Keywords:}

Blotto game

Incomplete Information

JEL classification:

C72

D72

\section{A B S T R A C T}

We consider a Blotto game with Incomplete Information. A pure-strategy symmetric monotonic Bayesian equilibrium is found and its properties are discussed.

(C) 2009 Elsevier B.V. All rights reserved.

\section{Introduction}

A topic of a particular interest in economics and political science is the behavior of players facing resource distribution decisions. We consider a Blotto game with Incomplete Information regarding player resources where players seek to maximize their expected payoffs. Players make allocation decisions simultaneously and whichever player spends the most on a particular contest wins that contest. The natural set of questions to ask in this situation is: How should players allocate their private budgets? Will all players compete for all prizes? Should we expect one player to win all the prizes? We find and characterize a pure-strategy symmetric monotonic Bayesian equilibrium (SMBE) for this game and answer these key questions.

There are two directions in the literature which are close to the topic of this paper. The first one is different Colonel Blotto games; see Borel (1921), Gross and Wagner (1950), Blackett (1958), Bellman (1969), Young (1978), Laslier and Picard (2002), Roberson (2006), Kvasov (2007), and Weinstein (2006) among others. In its most general case, the Blotto game presents two players with the problem of allocating a finite resource pool over $N$ "fronts" or contests, which are then played simultaneously. Each front or contest has its own valuation attached to it, and this in turn impacts how players choose to distribute their resources. Virtually all Blotto formulations are analyzed in a setting of complete information, where all players have common knowledge regarding their opponents' budget constraints. While analysis carried out under the assumption of complete information has been insightful,

\footnotetext{
论 We are grateful to an anonymous referee for helpful comments.

* Corresponding author. Department of Economics, University of Pittsburgh, Pittsburgh,

PA 15260, USA. Tel.: +1 412383 8156; fax: +1412 6481793.

E-mail address: alm75@pitt.edu (A. Matros).
}

the predictions of those papers are always mixed-strategy equilibria and flawless information about opponents, and in particular about opponents' budgetary constraints, is rarely available. ${ }^{1}$

A substantial body of literature exists on one- and multi-object auctions where bidders are subjected to budgetary constraints during the game (Che and Gale, 1998, 2003; Benoit and Krishna, 2001; Szentes and Rosenthal, 2003a,b). Such models correspond closely to the one that we are analyzing as players are forced to allocate scarce resources over multiple contests. As in the case of the Blotto literature, virtually all analysis of such auctions has been carried out under conditions of complete information. In one of the few models with an incomplete information setting analyzed by Che and Gale (1998), private information enters the picture through player valuations of one object, not player budgets as in our model. Furthermore, work by Pitchik (1995) in a scenario of incomplete information that extends to budget constraints is limited by the imposition of a one-dimensional bidder "type" which determines both bidder's valuation and budget.

Although our model does not allow for dynamic investigations of multi-unit cases such as those conducted by Benoit and Krishna (2001), it has the advantage of being more realistic insofar as bidders' budgets are private information and that this private information is not restricted by the imposition of a bidder type which also determines bidder valuations. In this sense, our model represents a case of the general multi-object auction; we model a $\mathrm{N}$-object auction with budget constraints, but with the caveat that such constraints are private information. All that a bidder knows about her opponent is the distribution from which her budget was drawn. Also our results can be complimented with results on the so-called Chopsticks auction obtained by Szentes and Rosenthal (2003a,b). Their

\footnotetext{
${ }^{1}$ Snyder (1989) and Matros (2007) find pure-strategy equilibria in stochastic versions of Blotto games.
} 
model specifically requires three objects in a two-player auction, but each player wants to win a "majority" of objects.

\section{The model}

There are $K$ risk-neutral players. Each player $k$ has her private budget $X^{k}$. We assume that each $X^{k}$ is independently and identically distributed on the interval $[0,1]$ according to the increasing distribution function $F$, where $F(0)=0, F(1)=1$. There are $N$ prizes. The value of prize $i$ is $W_{i}>0$ for all players and $\sum_{i=1}^{N} W_{i}=1$. Each player $k$ has to allocate her budget $X^{k}$ across all $N$ prizes. The players are competing for all $N$ prizes simultaneously. We assume that player $k$ wins prize $i$ if $x_{i}^{k}>\max \left\{x_{i}^{1}, \ldots, x_{i}^{k-1}, x_{i}^{k+1}, \ldots, x_{i}^{K}\right\}$, where $x_{i}^{j}$ is the budget allocation of player $j$ for prize $i$. We will use the upper index for the players and the lower index for the prizes. All players submit their budget allocations simultaneously. A pure strategy of player $k$ is a $N$-dimensional vector $\left(x_{1}^{k}, \ldots, x_{N}^{k}\right)$, such that $\sum_{j=1}^{N} x_{j}^{k}=X^{k}$.

Player $k$ obtains the following payoff

$I_{1}^{k} W_{1}+\ldots+I_{N}^{k} W_{N}$

where

$I_{i}^{k}= \begin{cases}1, & \text { if } x_{i}^{k}>\max \left\{x_{i}^{1}, \ldots, x_{i}^{k-1}, x_{i}^{k+1}, \ldots, x_{i}^{K}\right\}, \\ 1 / l, \text { if } x_{i}^{k}= & \max \left\{x_{i}^{1}, \ldots, x_{i}^{K}\right\} \text { and there are } l \text { such players, } \\ 0, & \text { if } x_{i}^{k}<\max \left\{x_{i}^{1}, \ldots, x_{i}^{k-1}, x_{i}^{k+1}, \ldots, x_{i}^{K}\right\} .\end{cases}$

A strategy for a player $k$ is a function $\beta^{k}=\left(\beta_{1}^{k}, \ldots \beta_{N}^{k}\right):[0,1] \rightarrow[0,1]^{N}$ which determines her budget allocation. Of course,

$\beta_{1}^{k}\left(x^{k}\right)+\ldots+\beta_{N}^{k}\left(x^{k}\right) \equiv \chi^{k}$

and

$\beta_{i}^{k} \geq 0$ for any $k=1, \ldots, K$ and $i=1, \ldots, N$.

\subsection{Pure-strategy symmetric monotonic Bayesian equilibrium}

We are looking for a pure-strategy symmetric monotonic Bayesian equilibrium (SMBE) - an equilibrium where all players follow the same strategy, $\left(\beta_{1}^{k}, \beta_{2}^{k}, \ldots, \beta_{N}^{k}\right)=\left(\beta_{1}, \beta_{2}, \ldots, \beta_{N}\right)$ for any $k=1, \ldots, K$, and if $x>y$, then $\beta_{i}(x)>\beta_{i}(y)$ for any prize $i=1, \ldots, N .^{2}$

Theorem 1. If $G \equiv F^{K-1}$ is concave, then $\beta^{*}(x)=\left(W_{1} x, W_{2} x, \ldots, W_{N} x\right)$ constitutes a unique SMBE.

Proof. Suppose that all players but player 1 follow the symmetric, increasing and differentiable strategy $\beta^{*}(x)=\left(W_{1} x, W_{2} x, \ldots, W_{N} x\right)$.

Suppose that player 1 receives a budget, $X^{1}=x$, to allocate. We want to determine the optimal allocation $\left(x_{1}^{1}, \ldots, x_{N}^{1}\right)$, s.t. $x_{1}^{1}+\ldots+x_{N}^{1}=x$. Then, player 1 solves the following maximization problem

$$
\begin{aligned}
& \max _{\begin{array}{l}
x_{1}^{1}, \ldots, x_{N}^{1} \\
\quad
\end{array}} W_{1} \operatorname{Pr}\left\{x_{1}^{1}>W_{1} X^{2}, \ldots, x_{1}^{1}>W_{1} X^{K}\right\} \\
& +\ldots+W_{N} \operatorname{Pr}\left\{x_{N}^{1}>W_{N} X^{2}, \ldots, x_{N}^{1}>W_{N} X^{K}\right\}, \\
& \text { s.t. } \sum_{j=1}^{N} x_{j}^{1}=x .
\end{aligned}
$$

\footnotetext{
${ }^{2}$ Our requirement is similar to Noldeke and Samuelson's (2003) "Net Viability" condition.
}

Denote

$T\left(x_{1}^{1}, \ldots, x_{N}^{1}\right) \equiv W_{1} G\left(\frac{x_{1}^{1}}{W_{1}}\right)+\cdots+W_{N} G\left(\frac{x_{N}^{1}}{W_{N}}\right)$.

Since we assume that the private budgets are distributed according to the distribution function $F$ on the interval $[0,1]$, the maximization problem (2)-(3) becomes

$\max T\left(x_{1}^{1}, \ldots, x_{N}^{1}\right)$,

$x_{1}^{1}, \ldots, x_{N}^{1}$

s.t. $\sum_{j=1}^{N} x_{j}^{1}=x$.

The first order conditions are

$G^{\prime}\left(\frac{x_{1}^{1}}{W_{1}}\right)=G^{\prime}\left(\frac{x_{2}^{1}}{W_{2}}\right)=\ldots=G^{\prime}\left(\frac{x_{N}^{1}}{W_{N}}\right)$,

Or

$\frac{x_{1}^{1}}{W_{1}}=\frac{x_{2}^{1}}{W_{2}}=\ldots=\frac{x_{N}^{1}}{W_{N}}$.

Therefore,

$x_{j}^{1}=W_{j} x$

In order to finish the proof of the theorem, we have to verify that $T$ $\left(x_{1}^{1}, \ldots, x_{N}^{1}\right)$ is a concave function. Since, by the assumption, function $G$ is concave, $T\left(x_{1}^{1}, \ldots, x_{N}^{1}\right)$ must be a concave function as a sum of concave functions. ${ }^{3}$

The following example illustrates Theorem 1.

Example. Suppose that $F(t)=t^{\frac{2}{3}}, W_{1}=.75$, and $W_{2}=.25$.

If $K=2$, the main condition of the theorem holds: $G(t)=t^{\frac{2}{3}}$ is concave and there exists a SMBE which is given by the function $\beta^{*}(x)=$ $(.75 x, .25 x)$.

If $K=3$, the main condition of the theorem is violated: $G(t)=t^{4}$ is convex.

Suppose that $x=.8$. Fig. 1 shows the expected payoff of a player as a function of her spending for prize 1 , given that her opponents allocate their budgets according to the rule $\beta^{*}$. The red solid (blue dotted) line corresponds to $K=2(K=3)$. It is clear from the graph that $\beta^{*}(x)=\left(W_{1} x, W_{2} x\right)$ does not constitutes a SMBE in $K=3$ case. $^{4}$

\subsection{Properties of the $S M B E$}

Since the SMBE is described in the previous subsection, we can discuss its properties now. These properties provide answers to the questions we posed in the Introduction section. The next three results follow from the structure of the SMBE.

Corollary 1. All players compete for all prizes in the SMBE.

Corollary 2. Each player spends more on more valuable prizes in the SMBE.

\footnotetext{
${ }^{3}$ This is a well-known result that appears in all of the standard optimization theory texts. For example, see p. 519 of Blume and Simon (1994).

${ }^{4}$ Note that $G^{\prime \prime}=(K-1) F^{K-3}\left[F f^{\prime}+(K-2) f^{2}\right]$. Hence, for any given function $F$, there exists $K_{0}$ such that $G^{\prime \prime}>0$ for any $K>K_{0}$, or the main condition of the theorem will be violated.
} 


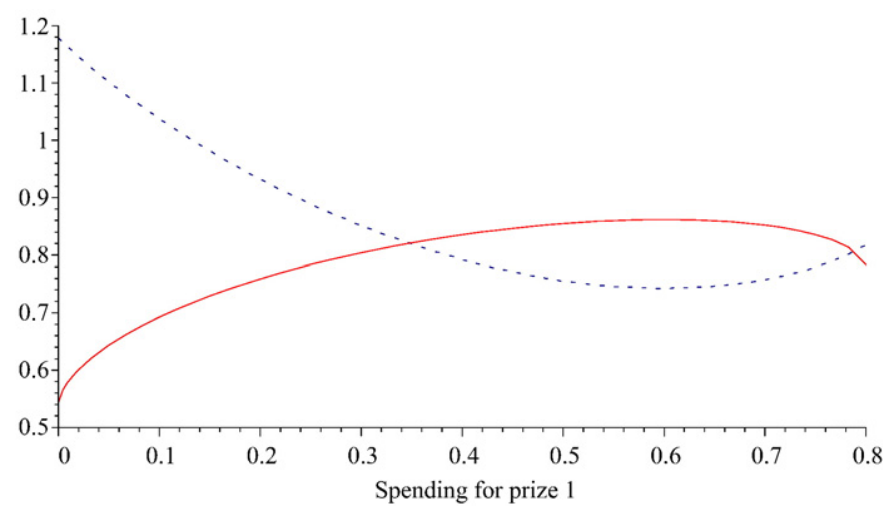

Fig. 1. Example.

Corollary 3. The player with the highest budget wins all prizes in the SMBE.

The expected equilibrium payoff of a player with budget $x$ can be calculated using the maximization problem (2) and (3). Corollary 2 provides an explanation for the result: a player either wins all prizes or none of them.

Corollary 4. The expected payoff of a player with budget $x$ is $G(x)$.
Benoit, Jean-Pierre, Krishna, Vijay, 2001. Multi-object auctions with budget constrained bidders. Review of Economic Studies 68, 155-179.

Blackett, D., 1958. Pure strategy solutions of Blotto games. Naval Research Logistics Quarterly 5, 107-110.

Blume, L., Simon, C., 1994. Mathematics for Economists. WW Norton.

Borel, E., 1921. “La Theorie de Jeu et les Equations Integrales a Noyan Symetrique.” Comptes Rendus de l'Academie des Sciences 173,1304-1308; English translation by L. Savage, "The theory of play and integral equations with skew symmetric kernels." Econometrica 21 (1953), 97-100.

Che, Yeon-Koo, Gale, Ian, 1998. Standard auctions with financially constrained bidders. Review of Economic Studies 65, 1-22.

Che, Yeon-Koo, Gale, Ian, 2003. Optimal design of research contests. American Economic Review 93, 646-671.

Gross, O., Wagner, R., 1950. A continuous Colonel Blotto game. RAND Corporation RM408.

Kvasov, D., 2007. Contests with limited resources. Journal of Economic Theory 136, 738-748.

Laslier, J., Picard, N., 2002. Distributive politics and electoral competition. Journal of Economic Theory 103, 106-130.

Matros, A. (2007). “Chinese Auctions." Mimeo, University of Pittsburgh.

Noldeke, Georg, Samuelson, Larry, 2003. Strategic choice handicaps when females seek high male net viability. Journal of Theoretical Biology 221, 53-59.

Pitchik, Carolyn. (1995). "Budget-Constrained Auctions with Incomplete Information." Mimeo, University of Toronto.

Roberson, B., 2006. The Colonel Blotto game. Economic Theory 29, 1-24.

Snyder, J., 1989. Election goals and the allocation of campaign resources. Econometrica 57, 637-660.

Szentes, B., Rosenthal, R., 2003a. Beyond chopsticks: symmetric equilibria in majority auction games. Games and Economic Behavior 45, 278-295.

Szentes, B., Rosenthal, R., 2003b. Three-object two-bidder simultaneous auctions: chopsticks and tetrahedral. Games and Economic Behavior 44, 114-133.

Weinstein, J. (2006). "Two Notes on the Blotto Game." Mimeo, MIT

Young, H.P., 1978. The allocation of funds in lobbying and campaigning. Behavioral Science 23, 21-31.

\section{References}

Bellman, Richard, 1969. Short notes on 'Colonel Blotto' and analogous games. SIAM

Review 11, 66-68. 